\title{
La escritura académica universitaria: diferentes perspectivas de estudio
}

DOI: $\underline{\text { https://doi.org/10.32870/dse.v0i11.275 }}$

Crisanto Salazar González*

Resumen: Este trabajo recupera las diferentes líneas de investigación que se han planteado para el estudio de la escritura académica en el contexto de la educación superior. A partir de la revisión de la literatura se identifican tres dimensiones de estudio de la escritura - lingüística, cognitiva y social- y sus correspondientes perspectivas o enfoques de investigación que exploran, analizan y explican la escritura académica que realizan los jóvenes en su formación universitaria. Así, este trabajo presenta la ubicación geográfica y temporal de estudio de la escritura, el planteamiento teórico de las dimensiones de estudio de la lengua escrita, las dimensiones y perspectivas de investigación de la escritura académica. Palabras clave: Escritura académica, prácticas de escritura, literacidad, dimensiones de la escritura, perspectivas de la escritura.

\begin{abstract}
This work recovers different research issues that had been raised on the study of academic writing in the higher education context. Three dimensions on the writing study - linguistic, cognitive and social- can be identified from the literature reviewed and their corresponding perspectives or research approaches that explore, analyze and explain how young students used academic writing in their university education development. Therefore, this work presents a study of the temporary and geographical location of writing, a theory approach on the dimensions of written language and research perspectives on academic writing. Key words: Academic writing, written practices, literacy, writing dimensions, written perspectives.
\end{abstract}

La escritura académica universitaria está siendo considerada como una nueva línea de investigación puesto que es objeto de estudio sistemático y de enseñanza desde hace dos décadas en el contexto anglosajón y una década en el ámbito latinoamericano. Algunos investigadores señalan que históricamente se ha priorizado la comprensión y producción textual en la educación básica debido a que leer y escribir ha sido la misión histórica de este nivel educativo (Ortiz, 2011: 18). Sin embargo, para Carlino (2005) la escritura es una de las actividades intelectuales más formativas que existen, no sólo en el nivel básico sino de todo el proceso educativo. De ahí que la escritura académica se coloca como un tema emergente de investigación de las instituciones y centros de investigación educativa.

En este sentido, la escritura académica adquiere una relevancia en el contexto de la investigación educativa a partir de diversas líneas de investigación que convergen en el propósito de explicar y comprender la escritura que desarrollan los jóvenes durante su proceso de formación profesional. El lenguaje escrito, según Parodi (2008), se presenta como un componente esencial para el aprendizaje en las disciplinas, ya sea por medio de la elaboración de escritos que van desde un resumen

\footnotetext{
* Estudiante del Doctorado en Educación de la Universidad de Guadalajara. Correo electrónico:crisantosalazar@hotmail.com
} 
hasta una tesis de grado o un artículo de investigación; aunque estos estudios sólo logran tocar la punta del icerberg de aquello que obstaculiza o limita el desarrollo de habilidades de escritura en los jóvenes profesionistas.

Así, este trabajo forma parte de la tesis de grado "La escritura académica en educación superior: una práctica social para la formación profesional", cuyo objetivo es analizar las prácticas letradas que caracterizan a la escritura académica en educación superior como una manera de comprender las situaciones en que se genera la práctica de la lengua escrita en una disciplina de estudio profesional. De ahí que este estudio aporte elementos para el estado de la cuestión en la temática de la escritura académica universitaria y tenga como propósito revisar y delimitar las distintas miradas que exploran, analizan y explican la escritura académica en el contexto universitario, con la idea de elaborar un esquema representativo de las diferentes posibilidades de abordar los problemas de escritura académica que caracterizan la formación universitaria.

Para esto se revisó lo publicado durante los últimos quince años sobre la escritura académica en educación superior, priorizando la temática de estudio, el objeto de estudio, las características de la investigación, las referencias teóricas y los estudios recientes. El procedimiento estuvo marcado de la siguiente manera: búsqueda de fuentes referidas a la escritura académica, clasificación de las fuentes, elaboración de fichas de trabajo, identificación y clasificación de los estudios, elaboración de esquemas, elaboración y revisión final. Así, este trabajo se presenta en cuatro momentos: ubicación geográfica y temporal de los estudios sobre la escritura académica, planteamiento teórico de las dimensiones de estudio de la lengua escrita, dimensiones y perspectivas de investigación de la escritura académica y las conclusiones.

\section{Ubicación temporal y geográfica de las últimas investigaciones}

La escritura académica universitaria es un tema de investigación sumamente nuevo en la lengua hispana. Carlino (2013) en su artículo "Alfabetización académica diez años después" da cuenta de la escasa literatura en español que existía en el año 2000 sobre la lectura y escritura en la universidad, mientras que en la lengua anglosajona abundaban las ediciones sobre esta temática. Al parecer, dice la autora, en el mundo hispano no existían estas investigaciones y si llegaron a existir se enfocaban únicamente en la enseñanza de la interpretación y producción textual, predominando los estudios lingüísticos y psicológicos centrados en el lector. Sin embargo, dada su persistencia y la de su equipo de investigadores, es a partir del 2001 que se instaura la lectura y escritura como objeto de enseñanza en las universidades argentinas, no como un asunto remedial sino como una responsabilidad de las instituciones educativas de compartir las prácticas letradas propias de cada disciplina profesional.

En esta radiografía está el trabajo que presentan López y Pérez (2013), Carrasco y Albarrán (2013) y López y Castillo (2013) en los Estados del conocimiento que publica el Consejo Mexicano de Investigación Educativa (COMIE). A partir de esta revisión se puede juzgar que México se 
incluye en esta temática de investigación con un corpus de 328 trabajos de investigación en diez años: 140 artículos de investigación, 17 trabajos de tesis de grado y, en menor número, la publicación de 15 libros insertados en los diferentes niveles de educación en México: básica, media básica, media superior, superior y posgrado, además de educación especial y educación para adultos. Incluso algunos investigadores se repiten tanto en artículos como en libros y en capítulos de libros. Además, no existe un criterio claro del orden temático, ya que los temas aparecen otra vez como subtemas de otros capítulos. Otro dato relevante que se observa de estas investigaciones es que se localizan, predominantemente, en la ciudad de México, Guadalajara, Puebla y Baja California.

El análisis de los Estados del conocimiento del COMIE, de acuerdo con López y Pérez (2013), Carrasco y Albarrán (2013) y López y Castillo (2013), sugiere tres puntos a destacar: a) Los estudios sobre la escritura y la lectura como prácticas sociales y situadas en construcción que comienzan a ser objeto de investigación desde una perspectiva social. b) El tema de la escritura y lectura aparece desarticulado entre los programas de los diferentes niveles educativos, y las actividades de enseñanza y aprendizaje son incompatibles con estos mismos programas. c) El lenguaje oral y escrito se aborda de manera multidisciplinaria y aislada para conocer el papel que tiene para el desarrollo social y educativo en distintas comunidades. Por lo que se puede juzgar, la investigación en esta temática es todavía insuficiente y, además, parte de iniciativas individuales que se ahogan en el enorme número de prioridades emergentes en el ámbito mexicano. De lo anterior se desprende que hace falta una mayor sistematización de los estudios sobre la lectura y escritura en el sistema educativo mexicano, situados en el contexto de formación universitaria.

Con una temática que se puede considerar longeva en el mundo hispano y los desatinos en el contexto mexicano, lo que hoy expongo constituye un mapa de las distintas miradas que conforman los estudios de la escritura académica. Para lograrlo, valen como referencias principales los estudios de Barton y Hamilton (1998), Castelló, Bañales y Vega (2010), Ortiz (2011) y Carlino (2013), ya que éstos aportan elementos importantes, en una u otra línea, para la configuración de las diferentes perspectivas de investigación en esta temática.

\section{Dimensiones de estudio de la lengua escrita}

Para algunos autores como Street (2009) y Kalman (2003), los estudios sobre el lenguaje escrito adquieren un papel preponderante a partir de los años ochenta. A partir de este periodo se realizan investigaciones que exploran las características que poseen los textos que se elaboran dentro de un ámbito o actividad social determinada, la relación que guardan estas características con el ambiente y cómo adquieren significados al retomar sus convenciones o normas como miembros de una comunidad práctica. En esta temporalidad Kalman señala:

A principios de los años ochenta antropólogos, sociolingüistas y psicólogos sociales iniciaban una agenda de investigación... Buscaban entender cómo se usa la lengua escrita, cómo la construimos socialmente, qué valor tiene en diferentes lugares y culturas. Se estudiaba la alfabetización como una práctica social (Kalman, 2003, p. 21). 
Por su parte, Street (2009) expone que en un principio, los procesos de escritura se explicaban a través de la teoría cognitiva, pero con los estudios lingüísticos y etnográficos, a partir de 1980, se adquiere una mayor comprensión del texto escrito como práctica social basada en el género. El mismo autor plantea que "La Teoría de Género reconoce que a) existe una gran variedad de comunidades discursivas con sus propias normas y convenciones para construir y debatir el conocimiento, y b) que los textos varían lingüísticamente de acuerdo a su propósito y contexto" (Street, 2009, p. 3).

Sin embargo, en esta misma revisión teórica se advierte la necesidad de considerar una perspectiva que no limite los alcances del discurso escrito, sino que lo complementen en todas sus posibilidades discursivas y significativas. Para obtener un mejor efecto, el análisis de la escritura académica se debe plantear desde una perspectiva que integre los distintos estudios del discurso escrito para una mayor comprensión, dada su complejidad y multiplicidad de significados (Bajtín, 2009; Van Dijk, 2000; Parodi, 2002 y Cassany, 2008).

Esta postura se explica en los siguientes planteamientos: Bajtín (2009) propone que la diversidad de los géneros discursivos es tan grande que no hay ni puede haber un solo enfoque para su estudio; Van Dijk (2000), por su parte, sugiere adoptar métodos de análisis distintos a los tradicionales y asumir un enfoque multidisciplinar en la escritura; en cambio, Parodi (2002) plantea una perspectiva integradora de las diferentes aproximaciones que se han ido llevando a cabo para su descripción; y Cassany (2008) remarca que la perspectiva sociocultural permite determinar los factores del contexto en que se da la práctica de la lectura y la escritura, como una característica de la práctica letrada contemporánea.

Con estos antecedentes, a partir del análisis del concepto de discurso, Van Dijk (2008) propone tres dimensiones para su estudio: a) el uso del lenguaje; b) la comunicación de creencias (cognición) y, c) la interacción en situaciones de índole social. De ahí que el estudio del lenguaje escrito puede ser objeto de estudio desde distintas disciplinas, como serían: la lingüística para el estudio específico del lenguaje y su utilización; la psicología para el estudio de las creencias y de cómo éstas se comunican; y las ciencias sociales para el análisis de las interacciones en situaciones sociales (Van Dijk, 2008: 23).

De acuerdo con estas orientaciones, las distintas aproximaciones de estudio de la lengua escrita se pueden categorizar tomando en cuenta el objeto del que se ocupan, como puede ser la forma de organización de la información, los procesos cognitivos implicados en la construcción e interpretación de la escritura y el contexto de interacción social en que se produce la escritura. Así pues, los diversos estudios e investigaciones sobre la escritura académica estarán enmarcados, en una primera instancia, en tres dimensiones de estudio: la dimensión lingüística, la dimensión cognitiva y la dimensión social. Mientras que en la segunda estarán las perspectivas o enfoques que se plantean desde las mismas dimensiones de estudio. 


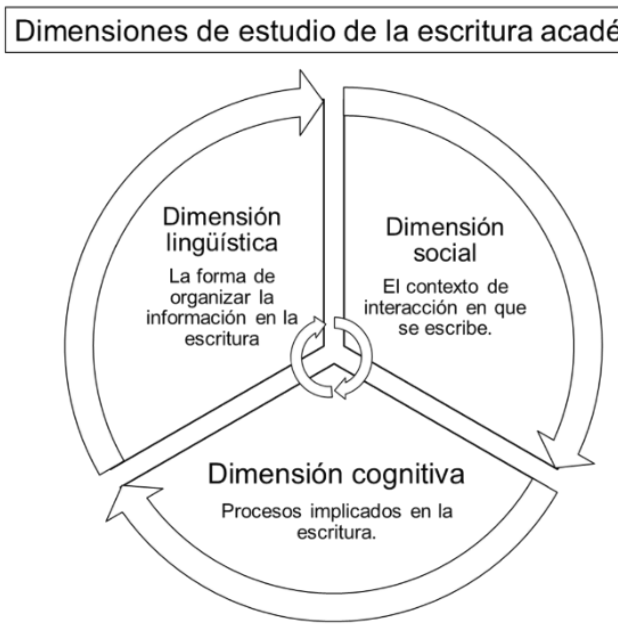

\section{Dimensión lingüística de la escritura académica}

La dimensión lingüística de la escritura tiene su origen en los estudios de la lengua y se fundamenta en las teorías gramaticales y discursivas. En su mayor parte, las investigaciones que se realizan desde esta dimensión toman como principal marco de referencia el análisis del discurso de Van Dijk (2008).

En esta dimensión, el objeto de estudio se centra en el análisis de los distintos elementos que permiten organizar la información en el discurso, como el propósito comunicativo del discurso, el tipo de texto, las relaciones entre segmentos de información, el planteamiento de las ideas u oraciones en una unidad temática y la conjunción y selección de palabras para construir una oración.

También, el uso de la lengua escrita está determinado por el ámbito académico o profesional en que ésta se produce y se caracteriza por cuatro niveles de especificidad lingüística, mismos que plantea Ferrero (2002): nivel léxico, nivel morfológico, nivel sintáctico y nivel del texto.

En el nivel léxico, Ferrero destaca el uso de términos propios del ejercicio de la profesión. Estos términos caracterizan un determinado campo del saber en relación con el sistema de redes conceptuales al que estos se remiten (Ferrero, 2002: 5).

En cambio, el nivel morfológico estudia los procesos de formación de palabras (derivación y composición) que caracterizan a cada tipo de actividad en la profesión.

Por su parte, en el nivel sintáctico destaca la nominalización de procedimientos habituales/ comunes en los textos especializados, propio de la formalidad del registro en una profesión.

$Y$ en cuanto al nivel textual sobresalen las partes prototípicas o de superestructura del discurso. Lo que en el ensayo tradicional se conoce como introducción, cuerpo o desarrollo y conclusión (Ferrero, 2002: 5-7). 
De ahí que todo escrito, de acuerdo con la forma en que se presenta y organiza la información, tiene un propósito específico para quien escribe y para quien lo lee. En esta idea Van Dijk (2008: 33) propone tomar en cuenta las reglas de cohesión y de coherencia textual. En la primera, un texto es cohesivo cuando las partes que integran al texto guardan relaciones de sentido y se refieren a la unidad interna del texto; en la segunda, el texto es coherente cuando existe una relación con el modo en que se organiza la información semántica. En este sentido se podría afirmar que la lingüística aporta elementos importantes para comprender los requisitos que debe cumplir el texto, es decir, cómo se debe presentar la información de acuerdo con el objetivo o función que pretende alcanzar en el lector a quien se dirige. En la consideración de esta serie de requisitos que debe cumplir el texto está que se debe dar la interacción/comunicación entre autor y lector.

El trabajo de Castro y Sánchez (2013) es un claro ejemplo de investigación en esta dimensión de la escritura académica. Ahí los autores realizan un análisis lingüístico de un corpus de cuarenta ensayos escolares, y por medio de la investigación cualitativa y del análisis del discurso exploran los recursos discursivos que utilizan los estudiantes en la construcción y expresión de opinión durante la formación académica. Para el análisis y descripción de los datos, los autores se plantean tres cuestiones: estructura del texto, inserción de la voz y construcción de perspectiva, mismos que se describen en los siguientes resultados.

En cuanto a la estructura del texto: a) los estudiantes conocen y manejan una sola estructura prototípica de organización y distribución de la información atribuibles al ensayo escolar (introducción, desarrollo y conclusión); y, b) los ensayos se caracterizan por una variación en la presencia o no de un título, la inclusión de epígrafes, la utilización de citas y/o referencias a lo largo de la exposición-argumentación, notas y, finalmente, la bibliografía consultada. En relación con la inserción y manejo de voces: a) los jóvenes desconocen las funciones retóricas que la cita cumple en el contexto disciplinar; $y, b$ ) las citas se incorporan de manera indirecta, es decir, el estudiante resume o sintetiza lo dicho por otro a través del recurso de la paráfrasis con el único objetivo de incorporar información. Mientras que en la construcción de perspectiva el estudiante tiende a las apreciaciones, cayendo en el dilema de ser objetivo y crítico a la vez, con una aparente contradicción en lo que a evaluación en el discurso especializado se refiere.

Como resultado de investigación, ante la falta de perspectiva Castro y Sánchez proponen fomentar un pensamiento complejo para que el estudiante desarrolle habilidades argumentativas que le permitan, entre otras cosas: considerar los marcos propios de la disciplina para la construcción de una opinión propia, aprender a pensar en situaciones abiertas de dialogo y discusión, investigar, informarse y evaluar los contenidos de aprendizaje, leer textos especializados para contrastar puntos de vista e identificar posturas similares o diferentes a las propias (Castro y Sánchez, 2013). A partir de esta investigación se identifican y se sugieren distintos aspectos que pueden ser objeto de estudio desde una dimensión lingüística de la escritura académica. 


\section{Dimensión cognitiva de la escritura académica}

En relación con la dimensión cognitiva, ésta nace en el campo de estudio de la psicología y se apoya en las teorías del desarrollo cognitivo. A diferencia de la dimensión lingüística, aquí, en términos de Castelló et al. (2010) y Ortiz (2011), el estudio no se centra en el discurso sino en el estudio de los procesos cognitivos que desarrolla el sujeto al momento de escribir, incluye los procesos metacognitivos de planeación, revisión y al conjunto de actividades que realiza el sujeto escritor para la detección y solución de problemas textuales en la escritura.

La psicología cognitiva es la que se ha dedicado especialmente al estudio de los diversos procesos y representaciones mentales que los usuarios del lenguaje realizan en la producción y comprensión del texto y de la conversación (Van Dijk, 2008, p. 42). Así, las investigaciones que se realizan desde una dimensión cognitiva presentan como objeto de estudio alguno de los procesos mentales internos que desarrolla el sujeto escritor, y es a través de la escritura que el sujeto manifiesta ese conjunto habilidades y competencias desarrolladas durante el proceso de elaboración de un texto.

Para Ortiz (2011), el principal marco de referencia en esta dimensión de estudio de la escritura académica se configura a partir de las investigaciones realizadas por Sommers (1978), Flower y Hayes (1981), Bereiter y Scardamalia (1987) y Cassany (1994). Además, en esta misma dimensión, algunos investigadores, como Nelms (1994) y Masiá (1996), destacan dos trabajos pioneros en esta línea de investigación: uno es de Rohman y Wlecke (1964) quienes identifican tres etapas del proceso de escritura — pre-escritura o descubrimiento de ideas, escritura y reescritura-, mismas que aún siguen vigentes en el campo de la enseñanza de la escritura (Masiá, 1996, p. 251). Otro es el modelo de escritura que propone Emig (1977), este modelo considera la escritura como un modo de aprender y, aún, tiene una influencia metodológica en los estudios de la escritura (Nelms, 1994, p. 122).

Sin embargo, tanto para Castelló et al. (2010) como para Ortiz (2011), con el modelo de Flower y Hayes (1981) se empezó a "enseñar" el proceso de composición escrita en las escuelas, con el fin de disminuir los problemas de escritura en los jóvenes. Para Flower y Hayes, el proceso de escritura se explica a partir de tres procesos: la planeación, la traducción y la revisión.

Primero, la planeación incluye tres subprocesos: generación, organización y establecimiento de metas. Cuya función es tomar información del ambiente de la tarea y de la memoria a largo plazo y usarla para establecer metas y un plan de escritura que guíe la producción de ideas en el texto. Luego, se realiza la traducción en función del plan de escritura para producir el lenguaje correspondiente, de acuerdo con la información, en la memoria del escritor. Por último, la revisión que incluye la calidad del texto producido en el proceso de traducción. Se corrige en relación con el lenguaje y las convenciones de contexto, así como la precisión del significado (Flower y Hayes, 1981). 
El modelo propuesto por Flower y Hayes, explica Ortiz (2011), concibe la composición escrita como una actividad recursiva, dirigida a la consecución de objetivos retóricos de la lengua escrita. Además, agrega, se ha criticado la universalidad y generalidad con que se concibe el proceso de escritura, dejando de lado otros aspectos, como el estado emocional de quien escribe y el contexto en que se produce el texto. De ahí que existan otras líneas de investigación que parten desde una dimensión cognitiva, como la perspectiva sociocognitiva que se revisa en la dimensión social de este texto.

Un texto relevante para destacar es el desarrollo de habilidades para la escritura eficiente, de Morles (2003). A partir de un enfoque heurístico, el autor integra a los teóricos e investigadores que han trabajado el tema de la escritura y propone un modelo operativo que describe el proceso de escritura en cuatro supuestos básicos:

Primero, explica Morles (2003), el modelo está dirigido sólo a personas relativamente maduras y con habilidades para la escritura. Retoma el modelo "transformar el conocimiento" de Bereiter, Burtis y Scardamalia (1988) y Bereiter y Scardamalia (1987). Segundo, parte del modelo de escritura planteado por Hayes (1996) y Flower y Hayes (1981), en cuanto a elaboración, traducción y revisión de la escritura. Tercero, resalta dos condiciones: a) el procesamiento de la escritura necesita de la memoria de largo plazo para almacenar la información y de la memoria de corto plazo para conducir los procesos cognitivos (Hayes, 1996), y b) escribir eficientemente requiere de las representaciones mentales, ya que el escritor imagina previamente aquello que desea escribir para expresarlo mediante el lenguaje (Schumacher y Ma., 1999). Y, cuarto, el escritor durante el proceso de escritura exhibe un estado de alerta, éste se explica en tres postulados: a) el proceso de escritura no es rígido, diría Cassany (1991); b) el escritor tiene conocimiento de las propiedades relevantes de la información y de los datos, expondría Flavell (1979); y c) el escritor es consciente de las diferentes etapas de su proceso de escritura, remarcaría Burón (1996).

Con esta propuesta Morles (2003) pretende superar las dificultades y deficiencias de escritura que tienen los estudiantes universitarios, en la idea de hacer del escritor una persona metacognitiva, consciente de sus procesos cognoscitivos y con capacidad de regular sus operaciones mentales cuando escribe. De acuerdo con este autor, la escritura se desarrolla como un proceso natural que se mejora en la medida en que ésta se va ejercitando y es en este ejercicio en el que se adquieren mayores habilidades para una escritura eficiente.

\section{Dimensión social de la escritura académica}

En cuanto a la dimensión social, se identifican cuatro perspectivas de estudio que parten de la idea de considerar a la lectura y escritura como una práctica social que se significa en el contexto social en el que interactúan tanto escritor como lector. Como principales perspectivas de estudio de la dimensión social se encuentran los nuevos estudios de literacidad, la perspectiva sociocognitiva, la perspectiva sociocultural y la de alfabetización académica, mismas que se exponen de manera sintética en los siguientes apartados. 


\section{Perspectiva de los nuevos estudios de literacidad}

En esta línea de la dimensión social se encuentran los nuevos estudios de literacidad, para lo que se retoma el planteamiento inicial de Barton y Hamilton: "Las literacidades están situadas. Todos los usos de la lengua escrita pueden ser vistos y encontrados en lugares y tiempos específicos. Igualmente, toda actividad letrada es indicio de amplias prácticas sociales" (Barton y Hamilton: 1998: 1). De ahí que la literacidad tenga su origen en las ciencias sociales, centrándose en los aspectos sociales y culturales del uso del lenguaje, y en su papel constitutivo en la vida social.

Los nuevos estudios de literacidad como perspectiva de investigación, de acuerdo con Gee (2000), hacen frente a los enfoques del comportamiento individual que predominaba en los años setenta en los estudios de la psicología conductista y de la psicología cognitivista, y abren paso a un enfoque centrado en el estudio de la lectura y escritura en la interacción social y cultural. Volviendo a Street (2009), los procesos de escritura se explicaban por medio de la teoría cognitiva, pero con los estudios lingüísticos y etnográficos el texto escrito adquiere una mayor comprensión como práctica social, por lo que se podría aseverar que la dimensión social de la escritura tiene su origen en esta perspectiva de los nuevos estudios de literacidad.

En cuanto al marco de referencia, las investigaciones que se realizan desde esta perspectiva tienen como marco común los estudios realizados por Gee (2000), Barton (1994), Street (2004) y Barton y Hamilton (1998), quienes plantean un enfoque desde la práctica social para significar y ampliar la comprensión de la lectura y escritura. De ahí que Gee (2000) defina a los nuevos estudios de literacidad como un movimiento que se conforma por una amplia variedad de disciplinas que, en las últimas décadas, han dado el "giro social" en los estudios de la lectura y escritura "lejos de un enfoque en las personas y las mentes 'privadas' a un enfoque en el conocimiento, las palabras y los hechos en sus contextos locales, sociales, culturales y políticos”, ya que la escritura sólo tiene sentido cuando se estudia en el contexto de las prácticas sociales y culturales (Gee, 2000: 177). 


\section{Dimensiones de estudio de la escritura académica}

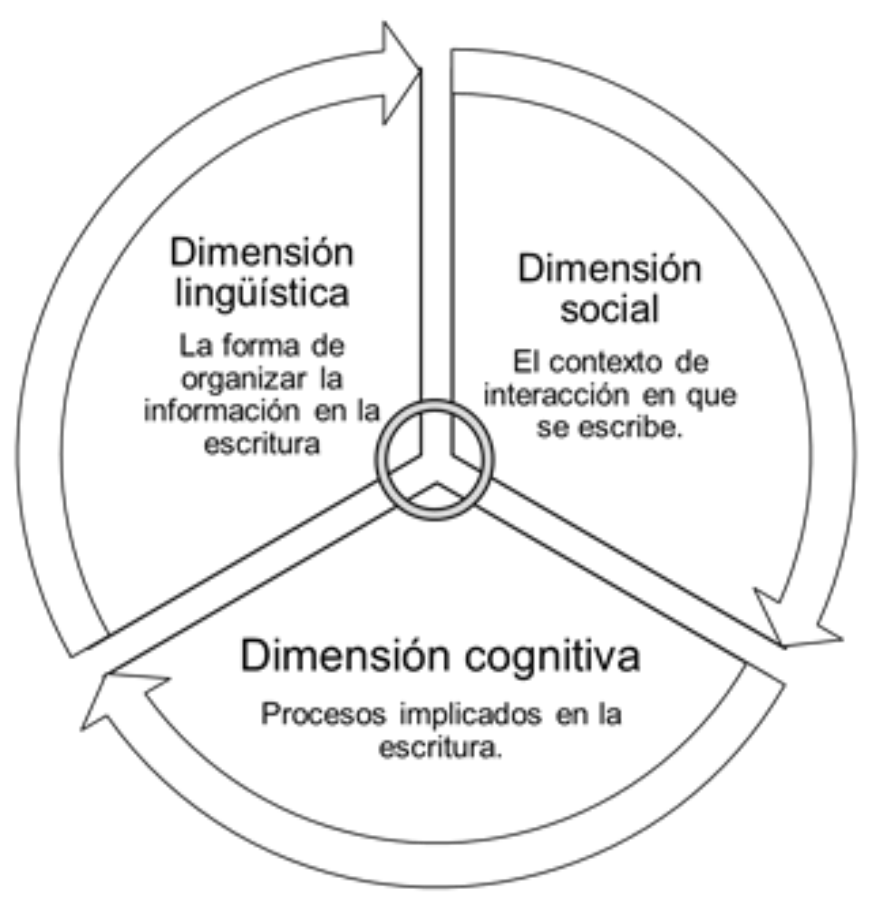

En esta perspectiva de los nuevos estudios de literacidad, dentro de la dimensión social predomina una mayor tradición en los estudios de la escritura y, además, se reconocen nuevas propuestas de estudio que abordan de manera muy específica los problemas de escritura en universidades de EE.UU., Australia y el Reino Unido. En esta temática resaltan los trabajos de Martin (2000), Bazerman (2004), Prior (1998) y Russell (1990 y 2002).

En el contexto hispano, el concepto de literacidad tiene sus controversias y desavenencias. Algunos autores lo manejan como propio y otros rastrean el concepto y hacen su propia interpretación en lengua española. En esta discusión, sumándose a López y Pérez (2013), participan Kalman (2003b), Ferreiro (2006b) y Carlino (2005), quienes a partir de la revisión de literatura especializada conceptualizan el término literacy, ya que éste no tiene una correspondencia en español, por lo que ha sido traducido de diversas maneras, como alfabetización, alfabetismo, escrituralidad, cultura escrita o literacidad. Para Ferreiro (2006b) el término literacidad, en inglés, hace referencia a las prácticas sociales de producción, circulación y uso de los textos escritos; mientras que en algunos estudios el concepto de alfabetización alude a procesos de decodificación. Kalman (2003b) admite que en español el término alfabetización hace referencia a un manejo rudimentario de la lengua y que literacidad "integra los usos de la lengua escrita, sus significados y las prácticas de leer y escribir" (como citó López y Pérez, 2013, p. 298). Por su parte, Carlino (2005) propone que 
el termino literacy o literacidad se puede entender como todo aquello que está organizado en torno a la cultura escrita en cualquier nivel educativo, pero también fuera del ámbito educacional en las diversas comunidades lectoras y escritoras. De aquí la amplitud que puede alcanzar esta perspectiva de estudio, no sólo en el contexto educativo sino en todos los ámbitos en los que se generan actividades donde la lectura y escritura de textos es parte fundamental, entre estos ámbitos están el social, el cultural, el laboral, el educativo y el recreativo, entre otros.

Un bello ejemplo de investigación en esta línea es el libro Escribir en la plaza, de Kalman (2003). Ahí, a través de la observación y la entrevista, la autora recopila datos sobre una variedad de eventos de lengua escrita que se llevan a cabo en la plaza de Santo Domingo de la ciudad de México entre personas que ejercen el oficio de escribanos. De acuerdo con la autora, este trabajo intenta contribuir a la creciente necesidad de comprender de una forma más amplia la cultura escrita y sus prácticas. Aporta a la construcción de un conocimiento más amplio acerca del fenómeno de la alfabetización y la presencia de la cultura escrita en la sociedad contemporánea (Kalman, 2003, pp. 23 y 24$)$.

\section{Perspectiva sociocognitiva}

En esta misma dimensión social, la perspectiva sociocognitiva se orienta hacia el estudio de la escritura como una práctica social y cultural, centrándose en el contexto y en todos aquellos aspectos sociales que inciden en la composición escrita. Así, el contexto forma parte significativa del proceso de producción de la escritura, mientras que la enseñanza se convierte en un aspecto fundamental que motiva el desarrollo de la misma.

Para Zimmerman y Risemberg (1997) "la escritura es más que la mera expresión escrita de una habilidad cognitiva: es un proceso social y cognitivo en donde los escritores... usan una variedad de métodos conductuales así como cognitivos para recabar y mantener experiencias afectivas y motivacionales" (citado en Castelló et al., 2010: 1261). De acuerdo con esta concepción la escritura adquiere una nueva dimensión de análisis que integra tanto los aspectos cognitivos de quien escribe como la situación de contexto en que se escribe.

Esta perspectiva, de acuerdo con Ortiz (2011: 24), se fundamenta desde los estudios y desde las aportaciones de diferentes disciplinas del conocimiento, como la antropología, con Gumperz y Hymes (1972), Petrucci (2003) y Rockwell (1982); la sociología, con Bourdieu (1980); la pragmática e historia, con A. Chartier (2004) y R. Chartier (2006); las teorías de la enunciación, con Bajtín (2009) y Benveniste (1978); y, el interaccionismo sociodiscursivo, con Bronckart (2004). De ahí que se generen propuestas de investigación sobre esta temática con enfoques muy particulares que se adaptan a la diversidad de la problemática de estudio de la escritura. En este caso se encuentran los estudios de regulación de la escritura y los estudios de las representaciones sociales de la escritura. 
Los estudios de regulación de la escritura desde la perspectiva sociocognitiva se caracterizan por utilizar procedimientos que se organizan en modelos que implican tanto estrategias de regulación cognitiva como estrategias afectivo-actitudinales. De acuerdo con Castelló et al. (2010), en esta posición se encuentran los modelos sociocognitivos de Zimmerman (2000) y de Graham y Harris (2009) quienes explican que la regulación de la escritura "enfatiza la importancia de varios factores cognitivos, conductuales, afectivos-motivacionales y ambientales que intervienen en toda actividad de escritura y que deben ser regulados a fin de lograr unos determinados objetivos" (Castelló, 2010: 1265).

En cambio, las representaciones sociales de la escritura, siguiendo a Ortiz (2011), se enfocan en el estudio de representación social que los sujetos - profesores y estudiantes - construyen sobre la escritura: es decir, las investigaciones se orientan al estudio de la representación que el escritor tiene respecto al tipo de texto, estructura, función, lector, etc. En esta línea de investigación se encuentran los estudios de Moscovici (1984), Abric (1994), Jodelet (1984), Bourdieu (1980) y R. Chartier (2000).

Asimismo, en esta línea se encuentran las investigaciones realizadas por García (2004), Carlino (2005b) y Peredo (2011) quienes, compartiendo la opinión de Ortiz (2011), identifican y analizan las representaciones sociales que profesores, estudiantes y profesionistas tienen sobre la lectura y la escritura en relación con la tarea y con los roles desempeñados en un contexto social determinado. Estos investigadores llegan a la conclusión de que la institución de cierta manera participa activamente en la construcción de las representaciones sociales, a través de los textos que ofrece para ser leídos, las consignas que propone, la evaluación que realiza y el tiempo que asigna para la práctica de la lectura y escritura.

\section{Perspectiva sociocultural}

En este escenario, la perspectiva sociocultural, en términos de Cassany (2008), tiene su origen en los estudios antropológicos y culturales, donde el significado del texto no está en el texto ni en el sujeto lector sino en el contexto social en el que interactúan tanto escritor como lector, en la comunidad cultural a la que pertenecen, y en el conocimiento sociocultural del grupo social humano al que se refiere el texto.

Desde esta perspectiva sociocultural, las investigaciones sobre la escritura académica centran su objeto de estudio en el conjunto de actividades que desarrolla el sujeto que escribe en el ámbito de una actividad específica. En términos de Kalman, "Los estudios socioculturales sobre el tema generalmente buscan describir y explicar los fenómenos relacionados con las prácticas de lengua escrita en situaciones específicas, los procesos de aprendizaje y apropiación de lectura y escritura" (Kalman, 2003, p. 41). Es decir, estas investigaciones se orientan al estudio de la condición social, económica y cultural del entorno que vive el sujeto, ya que éste afecta y significa a la producción escrita. 
En el marco de referencia, las investigaciones inscritas en esta perspectiva se sustentan en los trabajos desarrollados por Vygotsky (1995) acerca de la naturaleza del conocimiento, mismos que se abordan en las investigaciones pioneras de Scribner y Cole (1981). De acuerdo con Méndez (2004: 24), en estas investigaciones se considera a la lectura y escritura como producto de procesos psicológicos superiores que requieren de la autorregulación voluntaria, la realización consciente y el uso de signos para la mediación entre el texto que se escribe, el contexto en que se produce y la situación de quien escribe.

En el contexto mexicano, la perspectiva sociocultural se extendió durante la presente década a partir de los estudios sobre la apropiación de la cultura escrita de Rockwell (2000, 2006a), Kalman (2003b), entre otros, que fundamentan sus investigaciones en la teoría sociocultural y privilegian los procesos sociales del aprendizaje (Carrasco y Albarrán, 2013).

En abono a esta explicación, se asume que el lector juega un papel activo al producir su propio texto en relación con el contexto en que realiza la actividad de leer y el conjunto de normas que rigen la disciplina de aprendizaje universitario. En este sentido Peredo plantea que:

\footnotetext{
$\mathrm{Si}$ bien es cierto que el contexto y el orden institucional imponen la forma de lectura, eso no quiere decir que el lector es un receptor pasivo, sino que produce elaboraciones cognoscitivas complejas estableciendo una serie de vínculos entre la situación en la que lee, el texto y el contexto que le proporciona un texto estandarizado (Peredo, 2003, p. 21).
}

Con ello, el sujeto lector/escritor crea su propio texto, no un texto estandarizado sino un texto producto de su experiencia de vida, de sus conocimientos previos y de sus propias expectativas ante el texto objeto de aprendizaje y de producción, así como de la mediación que establece con el contexto y el espacio de formación educativa.

Entre los estudios más recientes, en esta perspectiva de investigación están los trabajos de Kalman (2003b), Englert, Mariage y Dunsmore (2006), Prior (2006) y Castelló (2007b) quienes han generado importantes contribuciones para la enseñanza de la regulación de la escritura en el nivel superior.

En el contexto mexicano sobre esta línea de investigación predominan los estudios de la lectura sobre la escritura, dejando entrever que uno y otro proceso se complementan. En este sentido Peredo (2001) caracteriza en su investigación el tipo de prácticas de lectura que manifiestan los estudiantes en los niveles de bachillerato, licenciatura y posgrado; además, identifica las habilidades que el estudiante desarrolla en cada nivel educativo, por ejemplo:

En el bachillerato la lectura se aleja de lo meramente informativo para dar paso a la lectura argumentativa y se caracteriza por ser reflexiva sobre una diversidad de temas, textos escolares y otros escritos. En este nivel el estudiante se apoya en el análisis, la crítica y la reflexión que aplica a los textos que se le proporcionan para su formación. 
En la licenciatura se observa la lectura técnica especializada del estudiante a través de habilidades y temas propios del campo de la profesión. Por lo regular, el estudiante hace uso de la memorización, la síntesis, la interpretación y la crítica de los textos que se le requieren y sugieren como parte de la profesión.

En posgrado, en cambio, la lectura se extiende hacia la sobre-especialización temática del estudiante. Aquí, el estudiante establece vínculos intertextuales a través del análisis, síntesis, crítica, razonamiento lógico y argumentativo de la lectura de referencias teóricas.

Así pues, las investigaciones que se inscriben en este ámbito tienden al estudio de aquellos aspectos que guían, regulan y concretizan el proceso de escritura, ya sea desde la óptica del maestro que promueve la escritura, del propio alumno en su necesidad de escribir e, incluso, desde la institución educativa.

\section{Perspectiva de alfabetización académica}

Desde una perspectiva de alfabetización académica, las investigaciones se orientan hacia el estudio de los procesos de enseñanza que se desarrollan para el aprendizaje de la escritura académica en el contexto educativo. Aquí destaca el concepto de alfabetización académica como "el proceso de enseñanza que puede (o no) ponerse en marcha para favorecer el acceso de los estudiantes a las diferentes culturas escritas de las disciplinas" (Carlino, 2013, p. 370).

A partir de esta concepción, Carlino (2013) sugiere como objeto de investigación, desde esta perspectiva, el conjunto de nociones y estrategias necesarias para participar en la cultura discursiva de las disciplinas, las actividades de producción y análisis de textos requeridos para aprender en la universidad, así como las prácticas de lenguaje y pensamiento propias del ámbito académico.

Como resultado de este tipo de investigaciones, señala Carlino (2013), se ha iniciado el proceso de inclusión de la lectura y escritura como parte de la formación universitaria en el contexto latinoamericano, mismo que afecta las tradiciones pedagógicas de la universidad y de todo un sistema educativo (Carlino, 2013: 370).

En esta perspectiva de investigación, desde la dimensión social, tiene como referencia las aportaciones de Bazerman (1988) y Curry y Lillis (2003) en un principio, continuando con las investigaciones de Benvegnú (2004) y Carlino (2005), quienes proponen un enfoque integrado o de enseñanza de la escritura en el contexto universitario desde el currículo y en la consideración de los múltiples factores que afectan el proceso educativo.

El artículo de Pérez y Rodríguez (2013) “¿Para qué se lee y se escribe en la universidad colombiana? Caracterización de prácticas de lectura y escritura en 17 universidades", se ubica en esta línea de investigación, ya que los autores describen el estado que guarda la práctica de la lectura y escritura en las universidades colombianas y sugieren algunas estrategias para atender los hallazgos encontrados en la investigación. De acuerdo con los autores, la investigación, desde un enfoque mixto, plantea un diseño de carácter descriptivo e interpretativo; se apoya en la observa- 
ción, la entrevista, el estudio de caso y el grupo de discusión, y trabaja con una muestra de 3715 estudiantes de 17 universidades, 17 grupos de discusión (estudiantes, docentes e investigadores) y 17 estudios de caso de maestros destacados.

A partir de la pregunta que da título al artículo, los resultados obtenidos se pueden resumir en cinco datos generales: a) No existe en las universidades documentación que dé cuenta de la lectura y escritura como directriz.; b) sólo $22 \%$ de los cursos de lectura y escritura se vinculan con las disciplinas y sólo se plantea como competencia general y no específica de la disciplina; c) en las clases predomina la lectura y escritura de los apuntes de clase sobre los otros tipos de textos, cuyo propósito principal es la evaluación y no la formación; d) los estudiantes manifiestan que "no se nos enseña a leer esos textos, es como si uno debiera saberlo, pero ni el colegio ni la universidad lo enseña"; e) en los estudios de caso, los maestros dicen promover la lectura y escritura por gusto, compromiso y como una manera de formar a los jóvenes. A partir de estos resultados, Pérez y Rodríguez (2013) demuestran la necesidad de atender las deficiencias de las prácticas de lectura y escritura, para ello proponen: establecer políticas para que la enseñanza de la lectura y escritura sea reconocida en la formación universitaria, rediseñar los programas de estudio orientados a la enseñanza de la lectura y la escritura como competencia de la disciplina profesional, promover en la práctica académica, la lectura y escritura de una variedad de textos de la disciplina de formación profesional.

\section{Dimensiones y perspectivas de estudio de la escritura académica}

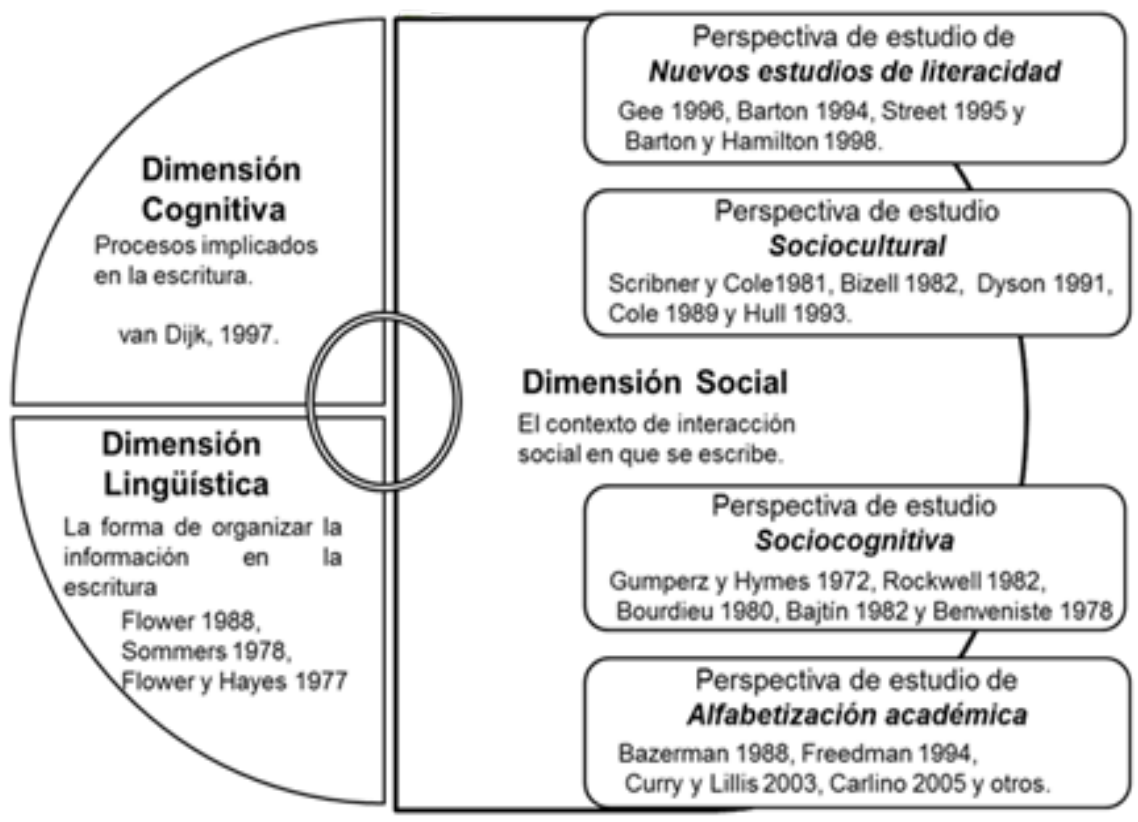


Sin bien el texto se presenta como un diagnóstico de la situación que guarda la lectura y escritura en las universidades colombianas, éste sugiere una transformación de las universidades a partir de colocar las prácticas de lectura y escritura como parte esencial de la formación de los estudiantes que se debe atender desde la disciplina de estudio en la universidad y no como algo adicional al conjunto de cursos planteados en el programa educativo.

\section{Conclusión}

Las diferentes líneas de investigación que tienen como propósito explicar y comprender la escritura académica universitaria convergen en tres grandes dimensiones de investigación: dimensión lingüística, dimensión cognitiva y dimensión social. Cada una de estas dimensiones está determinada por el objeto de estudio del que se ocupan, ya sea la forma de organización de la información en el texto, los procesos cognitivos implicados al momento de escribir y el contexto de interacción social en que el sujeto escritor desarrolla el ejercicio de escribir. Lo que significa que estas dimensiones no se contradicen sino que se complementan en un objetivo común de investigación, de ahí las diferentes perspectivas de estudio que combinan elementos de una y otra dimensión.

Como se expone, la escritura académica universitaria es una nueva línea de investigación que empezó a ser objeto de estudio sistemático y de enseñanza desde hace dos décadas y muy reciente en el ámbito latinoamericano. Esto no significa que no existan otras perspectivas de estudio, sino que éstas están orientadas al estudio de la escritura en educación básica y media, en las primeras letras y educación especial. Entre estos estudios están: la perspectiva psicolingüística de Emilia Ferreiro (2006b), la perspectiva neurolingüística, de Leal y Matute (2003), la perspectiva pedagógica en la que destaca Carrasco (2006a, 200b), la perspectiva sociohistórica con Barriga (2010) y los estudio de antropología cognitiva de Moore y Narciso (2011), que si bien no se desarrollan en estas páginas, son un importante marco de referencia en las investigaciones sobre la escritura académica universitaria.

Con ello se advierte que en el contexto universitario los docentes tienden a desarrollar más la parte lingüística de la producción escrita de los jóvenes, desdeñando la parte cognitiva y social de la escritura. En este escenario, los docentes se enfocan en la enseñanza y evaluación de cuestiones gramaticales y ortográficas, con el pretexto de que uno lleva a lo otro, sin cruzar la línea entre el plan de escritura que necesita desarrollar el estudiante y las condiciones de contexto que requiere para la disciplina.

Por lo tanto, la investigación sobre la escritura académica universitaria es tan amplia y compleja que se pierde en un mar de posibilidades discursivas que no puede quedarse sólo en la superficie, sino que requiere estudios orientados hacia la comprensión de las convenciones discursivas de la disciplina, de las habilidades de lectura y escritura propias del campo disciplinar y de la comunidad profesional donde se inserta la práctica de la lengua escrita. 


\section{Bibliografía}

Abric, J. (1994). Prácticas sociales y representaciones. México: Ediciones Coyoacán.

Ávila, A., A. Carrasco, A. Gómez, M. T. Guerra et al (coords.) (2013). Una de década de investigación educativa en conocimientos disciplinares en México. Matemáticas, ciencias naturales, lenguaje y lenguas extranjeras 2002-2010. México: ANUIES-COMIE.

Bajtín, M. M. (2009). Estética de la creación verbal. (Edición en ruso, 1979 y en español, 1982.) México: Siglo XXI.

Barriga, R. (2010). "Una hidra de siete cabezas y más: la enseñanza del español en el siglo XX mexicano”. En: Barriga Villanueva R. y P. Martín Butragueño (directores). Historia sociolingüística de México (vol. 2, pp. 1095-1194). México: El Colegio de México.

Barton, D. (1994). Literacy: An Introduction to the Ecology of written Language. Oxford: Blackwel.

y M. Hamilton (1998). Literacidades locales de lectura y escritura en una comunidad. Londres, Routledge (Taylor y Francis e-Library).

Bazerman, C. (1988). Shaping written knowledge. The genre and activity of the experimental article in science. Madison: University of Wisconsin Press.

(2004). "Intertextuality: How texts rely on other text". En: Bazerman, C. y P. Prior (eds.). What Writing Does and How It Does It. An Introduction to Analyzing Texts and Textual Practices. Mahwah, New Jersey: Lawrence Erlbaum Associates, pp. 83-96.

Benvegnú, M. A. (2004). "Las prácticas de lectura en la universidad: un taller para docentes”, en: P. Carlino (coord.), Leer y escribir en la universidad. Textos en Contexto núm. 6, Buenos Aires: Lectura y Vida.

Benveniste, É. (1978). Problemas de lingüística general. 2 vols. México: Siglo XXI.

Bereiter, C., J. Burtis, y M. Scardamalia (1988). "Cognitive operations in constructing main points in written composition". Journal of memory and language, 27(3), 261-278

y M. Scardamalia (1987). The psychology of written composition. Hillsdale, NJ: Lawrence Erlbaum Associates.

Bourdieu, P. (1980). El sentido práctico. Buenos Aires: Siglo XXI.

Bronckart, J. P. (2004). Actividad verbal, textos y discursos. Por un interaccionismo sociodiscursivo. Madrid: Fundación Infancia y Aprendizaje.

Burón, J. (1996). Enseñar a aprender: Introducción a la metacognición. Bilbao: Ediciones Mensajero.

Carlino, P. (2005). Escribir, leer y aprender en la universidad. Una introducción a la alfabetización académica. Buenos Aires: Fondo de Cultura Económica.

(2005b). "Representaciones sobre la escritura y formas de enseñarla en universidades de América del Norte". En: Revista de Educación, núm. 336, pp. 143-168. Disponible en: http:// www.revistaeducacion.mec.es/re336/re336_09.pdf 
(2013). “Alfabetización académica diez años después”. En: Revista Mexicana de Investigación Educativa, vol. 16, núm. 51, México, pp. 1227-1251 (ISSN: 14056666).

Carrasco, A. (2006a). Entre libros y estudiantes. México: Paidós.

— (2006b). "La lectura conquista adeptos en la escuela de educación básica". En: Vega L. (coord.). Alfabetización: retos y perspectivas. México: Facultad de Psicología de la UNAM. pp. 151-176.

— y C. Albarrán (2013). "Adquisición y desarrollo de la lengua escrita”. En: Ávila, A., A. Carrasco, A. Gómez, M. T. Guerra et al. (coords.). Una de década de investigación educativa en conocimientos disciplinares en México. Matemáticas, ciencias naturales, lenguaje y lenguas extranjeras 2002-2010. México: ANUIES-COMIE.

Cassany, D. (1991). Describir el escribir. Barcelona: Paidós.

M. Luna, y G. Sanz (1994). Enseñar lengua. Barcelona. Graó.

(2008). Prácticas letradas contemporáneas. México: Ríos de tintas.

Castelló, M. (2007a). "El proceso de composición de textos académicos". En: Castello, C., A. Iñesta, M. Miras, I. Solé, A. Teberosky y M. Zannoto (eds.). Escribir y comunicarse en contextos científicos y académicos. Conocimientos y estrategias. Barcelona: Grao (pp. 47-82).

G. Bañales, y N. Vega (2010). "Enfoques en la investigación de la regulación de escritura académica: Estado de la cuestión". En: Revista Electrónica de Investigación en Psicología de la Educación, vol. 8, núm. 22, diciembre. Almería y México: Universidad de Almería, pp.1253-1282.

Castro, M. C. y M. Sánchez (2013). "La expresión de opinión en textos académicos escritos por estudiantes universitarios". En: Revista Mexicana de Investigación Educativa, vol. 18, núm. 57. México, pp. 483-506.

Chartier, A. (2004). Enseñar a leer y a escribir. Una perspectiva histórica. México: Fondo de Cultura Económica.

Chartier, R. (2000). Las revoluciones de la cultura escrita. Barcelona: Gedisa.

Curry, M. J. y T. Lillis (2003). "Issues in academic writing in Higher Education". En: Coffin, C., M. J. Curry, A. Goodman Hewings, T. Lillis y J. Swan. Teaching academic Writing. Londres: Routlegde.

Emig, J. (1977) "Writing as a mode of learning". College Composition and Communication. 28, pp. 122-128.

Englert, C. S., T. V. Mariage y K. Dunsmore (2006). "Tenets of sociocultural Theory in Writing Instruction Research". En: Macarthur, C. A., S. Graham y J. Fitzgerald (eds.) Handbook of Writing Research. Nueva York: Guilford Press, pp. 208-221.

Ferrero, C. (2002). “Aproximación al análisis de los discursos profesionales”. Revista Signos, vol. 35, núm. 51-52. Valparaíso, s/pp. (ISSN 0718-0934).

Ferreiro, E. (2006b). "Librarians and basic education teachers in the context of 'Digital literacy". International Federation of Library Associations Journal, 31(1) 35-44. 
La escritura académica universitaria: diferentes perspectivas de estudio

Flavell, J. H. (1979) "Metacognition and cognition monitoring". American Psychologist. 34, pp. 906-911.

Flower, L. y J. R. Hayes (1981). "Teoría del proceso cognitivo de la escritura". College Composition and Communication. 32(4), pp. 365-387, http://doi.org/10.2307/356600.

García, M. (2004). “Aproximación al estudio de las representaciones de los docentes universitarios sobre el ensayo escolar". En: Revista de Teoría y Didáctica de las Ciencias Sociales, enerodiciembre, núm. 9. Mérida-Venezuela, pp. 9-34 (ISSN 1316-9505).

Gee, P. J. (2000). "Los Nuevos Estudios de Literacidad, 'socialmente situada' a la obra de lo social”. En: Barton, D. y M. Hamilton. Literacidad situada. La lectura y escritura en contexto. Londres y Nueva York: Routledge (L. Taylor y Francis e-Library).

Graham, S. y K. R. Harris (2009). "Almost 30 years of writing research: making sense of it all with "The Wrath oh khan"'. Learning disabilities research \& practice. 24 (2), pp. 58-68.

Gumperz, J. y D. Hymes (1972). Directions in Sociolinguistics. The Etnography of Communications. Nueva York: Holt, Rinehart and Winston.

Hayes, J. R. (1996) "A new framework for understanding cognition and affect in writing". En: Lewy, C. y S. Ransdell (eds.). The Science of Writing: Theories, Methods, Individual Differences, and Applications. Mahwah, NJ: Erlbaum, 1-27.

Jodelet, D. (1984). "La representación social: fenómenos, conceptos y teoría", en: S. Moscovici, Psicología Social II. Barcelona: Paidós, pp. 379-746.

Kalman, J. (2003). Escribir en la plaza. México: Fondo de Cultura Económica.

_ (2003b). "El acceso a la cultura escrita: la participación social y la apropiación de conocimientos en eventos cotidianos de lectura y escritura". Revista Mexicana de Investigación Educativa, vol. 8, núm. 17, enero-abril. México, pp. 37-66.

Leal, F. y E. Matute (2003). "La diversidad disciplinaria de los estudios sobre el lenguaje". En: Matute, E. y F. Leal (coords.). Introducción al estudio del español desde una perspectiva multidisciplinaria. Guadalajara: Universidad de Guadalajara, pp. 13-32.

López, M. L. y D. Castillo (2013). "Lenguaje y escuela: Perspectiva curricular", en: A. Ávila, A. Carrasco, A. Gómez, M. T. Guerra et al. (coord.). Una de década de investigación educativa en conocimientos disciplinares en México. Matemáticas, ciencias naturales, lenguaje y lenguas extranjeras 2002-2010. México: ANUIES-COMIE.

López, G. y C. Pérez (2013). "Discurso, cultura escrita y alfabetización: teorías, conceptos y comparación”. En: Ávila, A., A. Carrasco, A. Gómez, M. T. Guerra et al. (coords.). Una de década de investigación educativa en conocimientos disciplinares en México. Matemáticas, ciencias naturales, lenguaje y lenguas extranjeras 2002-2010. México: ANUIES-COMIE.

Martin, J. R. (2000). "Beyond exchange: Appraisal systems in English". En: Hunston, S. y G. Thompson (eds.). Evaluation in Text: Authorial Stance and the Construction of Discourse. Oxford: Oxford University Press, pp. 142-175. 
Masiá, M. L. (1996). "Traductores: traidores a su lengua materna". ELUA. Estudios de Lingüística, núm. 11. ISSN 0212-7636, pp. 251-260. http://hdl.handle.net/10045/6357.

Méndez, J. M. (2004). El escrito académico: un tejido de textos múltiples. México: Universidad Pedagógica Nacional.

Moore, P. y E. Narciso, (2011). "Modelos epistémicos de la lectura en estudiantes universitarios mexicanos". En: Revista Mexicana de Investigación Educativa, 16 (51), 1197-1225.

Morles, A. (2003). "Desarrollo de habilidades para la escritura eficiente". Lectura y vida. Universidad de Venezuela. Año 24, núm. 3. http://www.lecturayvida.fahce.unlp.edu.ar/numeros/ a24n3/24_03_Morles.pdf.

Moscovici, S. (1984). Psicología social II. Barcelona: Paidós.

Nelms, G. (1994). "Reevaluando Janet Emig el componer procesos de duodécimo grado: una perspectiva histórica". Rhetoric Review. 13 (1), pp. 108-130. Disponible: http://www.jstor.org/ stable/465782

Ortiz Casallas, E. M. (2011). "La escritura académica universitaria: estado del arte". İkala, Revista de Lenguaje y Cultura. 16 (28), Colombia, pp. 17-41.

Parodi, G. (2002). “Comprensión lingüística: ¿Hacia dónde vamos desde dónde estamos?”. En: Parodi, G. (ed.). Lingüística e Interdisciplinariedad: Desafios del nuevo milenio. Ensayos en honor a Marianne Peronard. Valparaíso: Ediciones Universitarias de Valparaíso, pp. 67-93. (2008). "Géneros discursivos y lengua escrita: propuesta de una concepción integral desde una perspectiva Sociocognitiva". En: Revista Letras, vol. 51, núm. 80. México, pp. 19-55.

Peredo M. A. (2001). "Las habilidades de lectura y la escolaridad". Perfiles Educativos, vol. 23, núm. 94. México, pp. 57-69.

_ (2003). "La importancia del contexto en la lectura laboral". Revista Mexicana de Investigación Educativa, vol. 8, núm. 17, enero-abril. México: COMIE, pp. 13-35.

(2011). "Representaciones docentes del déficit lector de los estudiantes". Revista Mexicana de Investigación Educativa, vol. 16, núm. 48, enero-marzo. México: COMIE, pp. 221-242 (ISSN: 14056666).

Pérez, M. y A. Rodríguez (2013). “¿Para qué se lee y se escribe en la universidad colombiana? Caracterización de prácticas de lectura y escritura en 17 universidades". En: Revista de Docencia Universitaria REDU. Número monográfico dedicado a Academic Writing. Vol. 11 (1), enero-abril, pp. 137-160.

Petrucci, A. (2003). La ciencia de la escritura. México: Fondo de Cultura Económica.

Prior, P. A. (1998). Writing/disciplinarity: A sociohistoric account of literate activity in the academy. Mahwah, NJ: Lawrence Erlbaum Associates.

Rockwell, E. (1982). "La relevancia de la etnografía para la transformación de la escuela". En: Memorias del Tercer Seminario Nacional de Investigaciones en Educación. Bogotá: Universidad Pedagógica Nacional, pp. 15-29. 
(2000). "La otra diversidad: historias múltiples de apropiación de la escritura". Divers Cité Langues. Vol. V. Disponible à http:// www.teluq.uquebec.ca/diverscite (2006a). "Apropiaciones indígenas de la escritura en tres dominios: religión, gobierno y escuela". En: Cultura Escrita y Sociedad, núm. 3, pp. 161- 218.

Russell, D. (1990). "Writing across the curriculum in historical perspective: Toward a social interpretation". En: College English, núm. 52, pp. 52-73.

— (2002). Writing in the academic disciplines. A curricular history. 2a ed., Carbondale y Edwardsville: Southern Illinois University Press.

Scribner, S. y M. Cole (1981). The psychology of literacy. Cambridge: Harvard University Press.

Schumacher, G. y Ma. C. (1999). "Representations in Writing: a Modularity Perspective", en: M. Torrance y D. Galbraith (eds). Knowing What to Write: Conceptual Processes in Text Production. Amsterdam: Amsterdam University Press.

Sommers, N. (1978). "Response to Sharon Crowley, components of the process". College Composition and Communication. 31, 378-388.

Street, B. (2004). "Los nuevos estudios de literacidad". En: Zavala, V., M. Niño-Murcia y P. Ames (eds.). Escritura y sociedad. Nuevas perspectivas teóricas y etnográficas. Lima: Pontificia Universidad Católica de Perú-Universidad del Pacífico, pp. 109-139.

— (2009). "Características ocultas de la escritura académica". (Documentos de Trabajo en la Educación lingüística). Vol. 24, núm. 1. King's College: WPEL.

Van Dijk, T. A. (2000). Estructuras y funciones del discurso. México: Siglo XXI.

- (2008). El discurso como estructura y proceso. Estudios sobre el discurso. Una introducción multidisciplinaria. Barcelona: Gedisa.

Vygotski, L. S. (1995). "Historia del desarrollo de las funciones psicológicas superiores", en: L. S. Vygotski, Obras escogidas, vol. III, Madrid, Visor (obra aparecida en ruso en 1931).

Zimmerman, B. J. (2000). "Attainment of self-regulation: A social cognitive perspective". En: Boekaerts, M., P. Pintrich y M. Zeidner (eds.). Handbook of self-regulation, research, and applications. Orlando: Academic Press, pp. 13-39.

— y R. Risemberg (1997). "Becoming a self-regulated writer: A social cognitive perspective". En: Contemporary educational psychology, núm. 22, pp. 73-101.

Recibido: 04/11/15

Dictaminado: 30/11/15

Corregido: 02/12/15

Aceptado: 04/12/15 